\title{
25 Research Suare \\ Investigation on Clocking Effect of Diffuser in a Multi-Stage Centrifugal Pump
}

\author{
Tan Ming-Gao \\ Jiangsu University \\ Sun Xue-Lei ( $\nabla 2665304236 @ q q . c o m$ ) \\ Jiangsu University https://orcid.org/0000-0002-5300-7463 \\ Wu Xian-Fang \\ Jiangsu University \\ Liu Hou-Lin \\ Jiangsu University
}

\section{Original Article}

Keywords: Multi-stage centrifugal pump, Diffuser, Clocking effect, Hydraulic performance, Pressure pulsation, Internal flow analysis

Posted Date: October 12th, 2021

DOI: https://doi.org/10.21203/rs.3.rs-955220/v1

License: (c) (1) This work is licensed under a Creative Commons Attribution 4.0 International License. Read Full License 


\section{Abstract}

The clocking effect is an important phenomenon in the multi-stage Rotating machinery. In order to master the rules and mechanism of diffuser clocking effect on the performance of multi-stage centrifugal pump, the orthogonal tests were applied to design the test scheme. The energy performance and outlet pressure pulsation of a multi-stage centrifugal pump with different diffuser clocking positions were synchronously measured. It was found that the diffuser clocking position had little influence on the energy performance, but had an obvious effect on the outlet pressure pulsation. When the diffuser clocking positions were $0^{\circ}$, $30^{\circ}, 30^{\circ}$ and $30^{\circ}$ (CL4), the effective value of outlet pressure pulsation and its amplitude at the main frequency (Impeller Rotation Frequency) were decreased the most, approximately $27.2 \%$ and $38.5 \%$ ,respectively. The CFD method was used to simulate the unsteady flow in the pump with the optimal diffuser clocking position (CL4) and without diffuser clocking position (CL1) respectively to reveal the mechanism of diffuser clocking effect. The simulation results showed that the change of diffuser clocking position can improve the inlet and outlet velocity distribution and reduce the area of high turbulent kinetic energy and the number of cores in the outlet flow passage, which is beneficial to the operation stability of the pump. Compared with the CL1, the hydraulic loss in the four diffusers was reduced by $2.43 \%, 12.15 \%, 11.43 \%$ and $13.19 \%$ respectively under the optimal diffuser clocking scheme (CL4), and the total reduction of hydraulic loss is about $1.11 \%$ of the pump head.

\section{Introduction}

Clocking effect refers to the phenomenon that the wake alteration of upstream components affects the fluid flow of downstream components by changing the circumferential position between rotor and static blades or between static and static blades[1-2]. The phenomenon can improve the performance of multistage rotating machinery and it is widely studied in pneumatic machinery [3-4].

In 1972, Walker[5] proposed the clocking effect for the first time by adjusting the circumferential position between the stators of a 1.5-stage compressor and found that the noise of the compressor was reduced. Saren et al.[6] studied the axial flow compressor clocking effect, and found that by changing the cascade clocking position, the efficiency of the unit could be slightly improved. Besides, it had an obvious influence on reducing the pressure load on the blades. Through experimental tests, Cizmas et al.[7] pointed out that the performance of the compression would also be affected by adjusting the circumferential position between rotor blades, and the clocking adjustment of rotor blades would improve the efficiency of the whole machine twice as much as that of the stationary blades. Hao et al.[8] studied the clocking effect of centrifugal compressor blades. She analyzed the changes of the aerodynamic load on the impeller surface under different clocking positions, and found the influence of the clocking effect of it. In addition, the influence was extended to the fatigue failure of the impeller blade structure.

Recently, the clocking effect has gradually become a research hotspot in the field of hydraulic machinery, and the clocking effect of multi-stage turbo-machinery can be used to optimize the performance of multistage pumps [9-10]. By adjusting the circumferential position of the components in the pump, the method 
takes into account the characteristics of the unsteady flow in the multistage pump, and improves the efficiency and stability without changing the pump structure. It has gradually become a key research direction to optimize the performance of multistage pumps [11-13].

Hong et al.[14] studied the inducer clocking position on the overall performance of the high-speed centrifugal pump through numerical simulation method. The results showed that the inducer clocking position affected the cavitation of the pump under different rotational speeds. With the flow rate increase, the influence of the inducer clocking position on cavitation increased gradually, and the occurrence position of cavitation also changed gradually. Fu et al. [15] simulated the impeller clocking effect on the multi-stage axial flow pump, and found that by changing the clocking position between the primary and the secondary impeller, the head and efficiency of the multi-stage pump were improved. Besides, the amplitude of pressure pulsation at the outlet of the primary impeller was significantly reduced. Ye et al. [16] took a high-head two-stage double-suction centrifugal pump as the research model. He studied the multi-stage pump clocking effect as well as the transient characteristics of the pump under different working conditions of $0.6 Q, 1.0 Q$ and $1.2 Q$ by designing four schemes. The results showed that the amplitude of pressure pulsation at the inlet tongue of the over-flow passage and the tongue of the pressurized water chamber were greatly weakened under different working conditions, and the radial force of the impeller changed periodically. Zhu et al.[17] studied the hydraulic performance of centrifugal pumps under different relative positions on diffusers and barrier tongues through experimental tests and numerical calculations. It was found that the relative positions of diffusers and barrier tongues had a great influence on the total energy loss inside the volute, but had little influence on the energy loss inside the diffusers and the work done by the impeller.

To summarize, many research studies have been carried out on the clocking effect of hydraulic machinery at present. However, most of the research is on the impeller clocking effect, and the research on the diffuser clocking effect of multi-stage pump is relatively few [18-20]. As an important over-flow component of multi-stage centrifugal pump, radial guide vane plays an important role in the performance of multi-stage pump[21-22]. In order to improve the working efficiency and stability of multi-stage centrifugal pump, a lot of research have been carried out on guide vane type selection and optimization, and the timing effect of guide vane is an urgent gap to be filled[23-24]. In this paper, the methods of combining experimental test and numerical simulation were used to study the diffuser clocking of a 5stage centrifugal pump and the mechanism of diffuser clocking. The results of this work can be used as reference for the research on multi-stage centrifugal pump performance optimization.

\section{Research Model}

A 5-stage centrifugal pump was chosen as the research model whose design flow rate, head and speed were $\mathrm{Q}=4.7 \mathrm{~m} 3 / \mathrm{h}, \mathrm{H}=38.6 \mathrm{~m}, \mathrm{n}=2950 \mathrm{r} / \mathrm{min}$. The structural parameters of the 5 impellers and the 4 diffusers working in the 5-stage centrifugal pump are the same. The physical picture of the pump is shown in Fig.1.(a) is the impeller and Fig.1.(b) is the diffuser. Table 1 summarizes the main parameters of impeller and diffuser. 
Table 1 Main Structural Parameters of Impeller and diffuser

\begin{tabular}{|lll|}
\hline Overcurrent component & Structural parameters & Numerical value \\
\hline Impeller & Impeller inlet diameter $\mathrm{d}_{1}(\mathrm{~mm})$ & 29 \\
\cline { 2 - 3 } & Impeller outlet diameter $\mathrm{d}_{2}(\mathrm{~mm})$ & 90 \\
\hline & Number of blades $\mathrm{z}$ & 8 \\
\hline & Blade exit angle $2\left(^{\circ}\right)$ & 41 \\
\hline Diffuser & Impeller outlet width $\mathrm{b}_{2}(\mathrm{~mm})$ & 2.6 \\
& Diameter of diffuser base circle $\mathrm{d}_{3}(\mathrm{~mm})$ & 30 \\
& Diffuser inlet width $\mathrm{b}_{3}(\mathrm{~mm})$ & 4 \\
& Number of diffuser blades z & 6 \\
& Diffuser outlet width $\mathrm{b}_{4}(\mathrm{~mm})$ & 4 \\
& Diffuser outlet diameter $\mathrm{d}_{4}(\mathrm{~mm})$ & 102 \\
\hline
\end{tabular}

\section{Test And Results}

To explore the influence of the diffuser clocking on the performance of the multi-stage centrifugal pump, 8 test schemes were designed through orthogonal test. What's more, the influence of diffuser clocking on the energy performance and pressure pulsation of the pump was studied through experimental test.

\subsection{Test Rig}

The multi-stage pump synchronous test experimental device is shown in Fig. 2. The test rig consisting of a water tank, an inlet valve, an outlet valve, a flowmeter, an inlet and outlet pressure transmitter, a vibration signal collector and sensor, a pressure pulsation signal collector and sensor, a multi-stage pump, a frequency conversion cabinet and the other parts are shown in Fig. 3.

\subsection{Data Acquisition System}

The data acquisition system including electromagnetic flowmeter, meter-explicit pressure transmitter, pressure pulsation sensor, three-phase electrical parameter acquisition instrument and rotational speed measuring instrument realized the synchronous acquisition of performance parameters. The performance parameters of the instrument required for system testing are summarized in Table 2. 
Table 2

Measuring Instruments and Performance Parameters for External Characteristics and Vibration Data of Multistage Pump

\begin{tabular}{|c|c|c|c|c|}
\hline Name of instrument & Model & Use & Range & Accuracy \\
\hline Electromagnetic flowmeter & $\begin{array}{l}\text { Shanghai Kent } \\
\text { KEFN-50-103- } \\
\text { G5E5 }\end{array}$ & $\begin{array}{l}\text { Measure pump } \\
\text { outlet flow }\end{array}$ & $0.71 \sim 70.65 \mathrm{~m}^{3} / \mathrm{h}$ & $\pm 0.3 \%$ \\
\hline $\begin{array}{l}\text { Meter-explicit pressure } \\
\text { transmitter }\end{array}$ & $\begin{array}{l}\text { Hangzhou Miko } \\
\text { MIK-3051-CP }\end{array}$ & $\begin{array}{l}\text { Measure pump } \\
\text { outlet pressure }\end{array}$ & 0-1Mpa & $0.075 \%$ \\
\hline Pressure pulsation sensor & $\begin{array}{l}\text { Chengdu Tester } \\
\text { CY301 }\end{array}$ & $\begin{array}{l}\text { Measure } \\
\text { pressure } \\
\text { pulsation }\end{array}$ & $\begin{array}{l}\text { Pressure } \\
0 \sim 600 \mathrm{KPa}\end{array}$ & $\pm 0.1 \%$ \\
\hline $\begin{array}{l}\text { Rotational speed measuring } \\
\text { instrument }\end{array}$ & $\begin{array}{l}\text { Xinbao } \\
\text { Technology } \\
\text { DT2234A }\end{array}$ & $\begin{array}{l}\text { Measure pump } \\
\text { speed }\end{array}$ & $2.5-99999 \mathrm{r} / \mathrm{min}$ & $0.1 \%$ \\
\hline $\begin{array}{l}\text { Three-phase electrical } \\
\text { parameter acquisition } \\
\text { instrument }\end{array}$ & TPA-3A & $\begin{array}{l}\text { Collect pump } \\
\text { motor input } \\
\text { power }\end{array}$ & -- & $\pm 2 \%$ \\
\hline
\end{tabular}

\subsection{Test Scheme}

Eight orthogonal experimental schemes were designed for the 5-stage centrifugal pump. Two adjacent pump stages were taken as a test factor, and the clockwise staggered angle of the secondary diffusers relative to the primary diffusers was passed for the level value of this test factor. Since the number of diffuser blades was 6 , two horizontal numbers were set, which were $0^{\circ}$ and $30^{\circ}$. Therefore, there are four horizontal factors included in the pump, namely, factor A, B, C and D. The orthogonal test table is summarized in Table 3.

Table 3

Test Scheme

\begin{tabular}{lllllllll} 
Test serial number & $\mathrm{CL} 1$ & $\mathrm{CL} 2$ & $\mathrm{CL3}$ & $\mathrm{CL} 4$ & $\mathrm{CL} 5$ & $\mathrm{CL6}$ & $\mathrm{CL7}$ & $\mathrm{CL8}$ \\
\hline $\mathrm{A}(12)$ & $0^{\circ}$ & $0^{\circ}$ & $0^{\circ}$ & $0^{\circ}$ & $30^{\circ}$ & $30^{\circ}$ & $30^{\circ}$ & $30^{\circ}$ \\
\hline $\mathrm{B}(23)$ & $0^{\circ}$ & $0^{\circ}$ & $30^{\circ}$ & $30^{\circ}$ & $0^{\circ}$ & $0^{\circ}$ & $30^{\circ}$ & $30^{\circ}$ \\
\hline $\mathrm{C}(34)$ & $0^{\circ}$ & $0^{\circ}$ & $30^{\circ}$ & $30^{\circ}$ & $30^{\circ}$ & $30^{\circ}$ & $0^{\circ}$ & $0^{\circ}$ \\
\hline $\mathrm{D}(45)$ & $0^{\circ}$ & $30^{\circ}$ & $0^{\circ}$ & $30^{\circ}$ & $0^{\circ}$ & $30^{\circ}$ & $0^{\circ}$ & $30^{\circ}$
\end{tabular}

The pressure pulsation monitoring point at the outlet of multistage pump arranged at twice the pipe diameter at the outlet of multistage pump, as shown in Fig. 4.

\subsection{Test Results}


A comparison chart of the head and efficiency of multi-stage pump under design flow rate is shown in Fig. 5. The dimensionless comparison method was adopted which dimensionless parameters $H / H_{\mathrm{CL} 1}$ and $\eta / \eta_{\mathrm{CL} 1}$ were used to characterize the change rate of the head and efficiency of each diffuser clocking scheme relative to the diffuser clocking scheme CL1.

As can be observed from Fig. 5, the scheme CL6 is the best one to improve the multi-stage pump external characteristics. The pump head and efficiency reach the maximum of the eight schemes, which are about $100.4 \%$ and $100.5 \%$ respectively of the scheme CL1. It can be observed that the diffuser clocking has little influence on the pump head and efficiency.

Figure 6 shows a time domain diagram of the pump outlet pressure coefficient in one cycle under different diffuser clocking schemes.

The outlet pressure pulsation curve under each diffuser clocking scheme fluctuates within the same range, and the periodicity of this is not obvious. The outlet pressure coefficient of the clocking scheme CL1 reaches the maximum value of about 0.032 at $0.4 \mathrm{~T}$. In addition, the maximum values of outlet pressure coefficient under the scheme CL2 to CL8 are lower than that under the clocking scheme CL1.

Further, the arithmetic root mean square of pressure coefficient is taken as the effective evaluation standard of the outlet pressure pulsation, and the calculation formula is as follows:

$$
\begin{gathered}
\mathrm{U}_{\mathrm{p}}=\sqrt{\frac{\sum_{\mathrm{i}=1}^{\mathrm{N}}\left(\mathrm{C}_{\mathrm{p}}(\mathrm{i})-\overline{\mathrm{C}_{\mathrm{p}}}\right)^{2}}{\mathrm{~N}}} \\
\overline{\mathrm{C}_{\mathrm{p}}}=\frac{1}{\mathrm{~N}} \sum_{\mathrm{i}=1}^{\mathrm{N}} \mathrm{C}_{\mathrm{p}}(\mathrm{i})
\end{gathered}
$$

The mean square root, the instantaneous value, the average value and the number of values of the pressure coefficient are Up, $\mathrm{Cp}(\mathrm{i})$, and, respectively.

The arithmetic root mean squares of pressure coefficients of each scheme are compared, as shown in Fig. 7.

Based on Fig. 7, the diffuser clocking effect has a significant influence on the pressure pulsation at the pump outlet. Compared with the CL1, the effective values of pressure coefficients under each diffuser clocking position are decreased to a certain extent, with ranges from 3.5-27.2\%. Besides, the root mean square decreases of pressure pulsation in the CL3 to CL8 are $10 \%$ more than the CL1, which have a significant impact on improving the stability of multi-stage pump outflow. Moreover, it can be presented 
that the clocking effect of the CL4 is the most obvious than others, and the effective value of outlet pressure pulsation is decreased by $27.2 \%$.

Figure 8 presents a frequency domain distribution figure of pressure coefficient. As can be observed from the Fig. 8, the distribution of outlet pressure coefficients is basically the same in each diffuser clocking scheme. Furthermore, the main frequency is the axis frequency (1IRF). This explains the outlet pressure pulsation frequency domain distributions are not changed owing to different diffuser clocking schemes. It suggests that the outlet pressure pulsation is still mainly affected by the rotation of the rotating shaft, but the main frequency amplitudes of pressure pulsation are varied under different diffuser clocking schemes.

Under different schemes, the amplitudes of the outlet pressure coefficient at the shaft frequency are shown in Fig. 9. In all schemes, the axial frequency amplitude under the CL1 is the largest, because all diffuser circumferential positions are the same under this scheme. Therefore, the axial frequency amplitude generated by each pump stage will be conducted to the next pump stage and it will produce cumulative effects. However, the other seven schemes all have different circumferential positions between one or more diffusers of previous pump stages, which leads to certain losses in the fluctuation phenomenon that the transmission shaft frequency is the main frequency. This explains that the amplitude of the shaft frequency and the effective value of the pressure coefficient are reduced. It makes the outlet flow more stable.

Corresponding to the greatest decrease on the effective value of the pressure coefficient of the CL4, the amplitude of the diffuser clocking scheme CL4 has the biggest change. Meanwhile, the amplitude of the CL4 is $35.8 \%$ less than the CL1, indicating that the scheme CL4 is the better one.

Since the amplitude of the main vibration frequency at the outlet of the multistage pump is more than that at the inlet, the clocking scheme CL4 is the most significant to improve the operation stability of the multi-stage pump, and the amplitude at the main frequency is reduced by $35.8 \%$, which shows that the vibration at the outlet of the multi-stage pump caused by the rotation of the rotating shaft under this scheme is greatly suppressed. Therefore, the CL4 is the best one to improve the operation stability of the multi-stage pump among the eight schemes.

\section{Numerical Simulation And Results}

To further analyze the internal flow mechanism of the diffuser clocking, CFD software is used to numerically simulate the internal flow of the pump under the optimal diffuser clocking scheme (CL4) and the scheme (CL1).

\subsection{Meshing Division}

ANSYS-ICEM software is used to mesh each computational fluid domain respectively. The computational domain divided by structured meshing is shown in Fig. 10, including impellers and pump chambers. The 
calculation domain divided by unstructured grid is shown in Fig. 11, including the first pump chamber, the diffusers and the last water pressure chamber.

\subsection{Boundary Conditions}

CFX software is used to carry out the steady-state calculation of the steady-state process of segmental multi-stage centrifugal pump, and the results are taken as the initial conditions of unsteady simulation for unsteady calculation. In the process of steady calculation, the impeller water body is set to Rotating, and the rest water body is set to Stationary. The Frozen Rotor model is adopted for the connection between the rotating domain and the stationary domain. Besides, the GGI model is adopted for the mesh association model. In the process of unsteady calculation, the connection model between the rotating domain and the stationary domain is transformed to the transient rotor/stator interface. The grid connection mode is kept to the GGI model.

RNG $k-\varepsilon$ turbulence model is used to simulate the full flow field of multi-stage centrifugal pump because it is reasonable[24]. In the calculation, the boundary combination of total pressure at the inlet and mass flow at the outlet is adopted. The specific setting is as follows: the inlet is $0 \mathrm{~Pa}$, and the mass flow at the outlet is set according to different working conditions of the multi-stage pump. The interface between the water body and the wall surface of the multi-stage pump is set as No Slip Wall.

In the process of solving the convective discretization of the governing equation, the second-order High Resolution Discrimination scheme is adopted, and the average residual value is set as the convergence criterion, with the convergence accuracy of $10^{-4}$. In the unsteady calculation, the time step is set to $\Delta t\left(\Delta t=5.65 \times 10^{-5} \mathrm{~s}\right)$. The impeller is calculated once every $1^{\circ}$ rotation, with a total of 3600 steps. Take the data of the last circle as the valid data for analysis.

\subsection{Numerical Simulation Results}

A comparison $\left(\varphi=0^{\circ}\right)$ between the simulated predicted value and the test value of the head and efficiency is shown in Fig. 12.

From Fig. 12 (a), it can be found that under low flow rate, the simulation value of the head is slightly lower than the test value of it. At working point of $3 \mathrm{~m}^{3} / \mathrm{h}$, the head simulation value is the closest to the test value. While under the large flow rate, the head simulation value is also slightly lower than the test value at $5 \mathrm{~m}^{3} / \mathrm{h}$ working point. The head prediction and the test values are $35.4 \mathrm{~m}$ and $36.7 \mathrm{~m}$ respectively, whereas that the difference between each working point is the largest, with a relative deviation of $3.54 \%$. At the rated operating point of $4.7 \mathrm{~m}^{3} / \mathrm{h}$, the head prediction and the test values are $35.7 \mathrm{~m}$ and $36.9 \mathrm{~m}$ respectively, with the relative deviation $3.25 \%$.

From Fig. 12 (b), it can be found that the trend of the predicted efficiency with the flow rate is consistent with the test results, and both increase first and then decrease with the flow rate increase. Under the full flow rate, the difference between the predicted efficiency value and the test value is the largest at the working point of $3 \mathrm{~m}^{3} / \mathrm{h}$, with the values of $46.1 \%$ and $44.2 \%$ respectively and a deviation of 1.9 
percentage points. At the rated operating point of $4.7 \mathrm{~m}^{3} / \mathrm{h}$, the efficiency prediction value and the test value are $53.6 \%$ and $53.0 \%$, respectively, and the deviation of them is 0.6 percentage points.

Under full flow rate, the predicted values of the head and efficiency of the pump obtained by simulation are closed to the test values of the pump. Besides, the two values are consistent with the trend of flow rate. It means that the numerical simulation calculation method is basically reliable.

\subsection{Analysis of Internal Flow of Multistage Pump Axial Plane}

\subsubsection{Internal Flow Analysis of Whole Pump}

The static pressure distributions on the axial surface of the multi-stage pump under the two schemes are shown in Fig. 13. The hydrostatic pressure of the pump is continuously rising from the inlet to the outlet. There is an obvious interface between high and low pressure areas in the impeller passage. In the same time, there is no obvious interface between high and low pressure areas in the diffuser passage. Under the two schemes, the circumferential positions of the diffusers of the first two pump stages are the same, as to the static pressure distribution in the flow passage basically.

In the clocking scheme CL4, the diffusers of the latter three pump stages are arranged in a continuous circumferential staggered manner. When the fluid flows out from the end of the impeller passage of the fifth pump stage to the outlet of the multi-stage pump, the high-pressure area of the pressurized water chamber is obviously expanded, and the pump chamber at the outlet of the fifth stage is also filled with the high-pressure area. It shows that the diffuser clocking of the multi-stage pump has significant effect. The CL4 increases the static pressure value at the outlet of the multi-stage pump and the head of the pump to a certain extent.

\subsubsection{Flow Analysis in Pump Cavity}

Figure 14 shows the graph of axial velocity distributions and streamlines of each pump stage chamber under the two schemes.

In the first pump stage, there are counter clockwise vortices in the pump cavity on the left side of the front cover plate of the diffuser, and the area of the vortices is basically the same. In the last four pump stages, there is a high-speed outlet area at the outlet of the impeller. There are two vortices with opposite rotation directions on both sides of the outlet area. The left vortex rotates counterclockwise and the right vortex rotates clockwise.

For $\mathrm{CL} 1$, the vortex sizes of the two outlets of the last four pump stage impellers are basically the same. The left vortex is closer to the rear cover plate of the front stage diffuser as well as the right vortex and the left vortex are on the same horizontal line. For CL4, the vortices on both sides of the impeller outlets of the last four pump stages have changed. It can be observed that the vortices on the left side of the impeller outlets of the third pump stage and the fifth pump stage are closer to the outer volute of the 
pump than before, whereas that the vortices on both sides are no longer on the same horizontal line. At the same time, the vortex core areas of the two vortices at the impeller outlets of the fourth pump stage are obviously reduced, and the impeller outflow is smoother.

\subsubsection{Internal Flow Analysis of diffuser Outlet Section}

Figure 15 shows the velocity distribution and streamline of the outlet section of each pump stage diffuser under the two schemes. It can be seen that after the fluid passing through the diffuser flow passage of each pump stage, there are 6 relatively high-speed zones with similar shapes and different areas on the outlet cross section of the diffuser. The velocity of certain fluids is relatively high, and the velocity distribution on the outlet cross section is uneven. From the streamline of the outlet section of the diffuser, it is found that the fluid will rotate counter clockwise before entering the next impeller.

From the comparison of the CL1 and the CL4, it can be found that the diffuser clocking of the first two pump stages is the same under the two schemes and that is why the velocity distribution at the outlet section of the diffuser of the first pump stage and the second pump stage is basically the same. From the comparison of the last three diffuser outlet sections, it can be found that under the CL4, the area of the relative high-speed zone on the outlet sections of the pump stage is obviously reduced. What's more, the corresponding maximum velocity is reduced, which shows that the diffusers circumferential staggered arrangement of the four pump stage is conducive to eliminating the uneven of velocity distribution at the outlet position of each diffuser flow channel and reducing the outlet flow rate of the diffuser. At the same time, it improves the effect of reducing speed and pressurizing the diffuser.

\subsubsection{Analysis of Internal Flow at Outlet of Multistage Pump}

The velocity distribution in the multi-stage pump on the outlet plane under the two schemes is Fig. 16.

From Fig. 16, it can be seen that the flow situation in the outlet axial plane under the two schemes is similar. There is a high-speed zone on the left side of the outlet channel. There are two whirlpools with different sizes and rotating clockwise in the outlet channel. The small whirlpool is located near the connection between the outlet channel and the water pressure chamber, whereas the large whirlpools are far away from the water pressure chamber and close to the right side of the outlet channel.

Compared with the outlet plane velocity distribution under the scheme CL1, the area of the high-speed zone near the left side of the outlet channel in the scheme CL4 is reduced, and the area of the vortex on the right side of the outlet channel is also smaller, which alleviates the blockage of the outlet flow of the outlet channel. This shows that the scheme CL4 effectively optimizes the outlet disorder of the multistage pump. The outlet flow of the pump is smoother.

\subsection{Hydraulic Loss Analysis of diffuser}

The hydraulic losses of the diffuser of each pump stage under the scheme CL1 and CL4 are shown in Fig. 17. 
As can be observed from Fig. 17, the hydraulic loss of diffuser flow passage of each pump stage in the scheme CL4 is reduced compared with that in the scheme CL1. From the first pump stage diffuser to the fourth pump stage diffuser, the reduced hydraulic loss is $0.0244 \mathrm{~m}, 0.132 \mathrm{~m}, 0.1276 \mathrm{~m}$ and $0.1432 \mathrm{~m}$ respectively, accounting for $2.43 \%, 12.15 \%, 11.43 \%$ and $13.19 \%$ of the hydraulic loss of diffuser of each pump stage respectively. Among them, it can be found that the hydraulic loss in the second to the fourth pump stages has reached more than $10 \%$ of its own hydraulic loss. The total hydraulic loss of the five pump stage has decreased by $0.4272 \mathrm{~m}$, accounting for $1.11 \%$ of the pump head.

\subsection{Analysis of Turbulent Kinetic Energy}

The turbulent kinetic energy distributions on the outlet shaft of the multi-stage pump in the scheme CL1 and CL4 are Fig. 18.

As can be observed from Fig. 18, on the outlet of the pump in the two schemes, the turbulent kinetic energy value in the pressurized water chamber is relatively small, within $0.77 \mathrm{~m}^{2} \mathrm{~s}^{-2}$. The high turbulent kinetic energy area mainly occurs in the outlet channel, with the maximum $3.2 \mathrm{~m} 2 \mathrm{~s}-2$.

In the scheme CL1, there are two cores with different sizes in the high turbulent kinetic energy area, and the area is larger. In the scheme CL4, the area of the high turbulent kinetic energy area at the outlet of the multi-stage pump tends to extend to the pressurized water chamber area and the outlet. At the same time, the core in the high turbulent kinetic energy area merges into one and the area of it is obviously reduced. This shows that the turbulent kinetic energy at the outlet of the multi-stage pump is reduced and the flow pattern is more stable in the scheme CL4.

\section{Conclusions}

The effect of diffuser clocking of multi-stage centrifugal pump is numerically analyzed through experimental tests and the CFD numerical simulation. Moreover, the internal flow rule in the multi-stage centrifugal pump is analyzed. The main conclusions are as follows:

(1) By adjusting the circumferential position between diffusers, it was found that the clocking of diffusers had limited influence on the head and efficiency of the pump, and the maximum change rate was only about $0.5 \%$. The diffuser clocking had a significant effect on the pump outlet pressure pulsation. Compared with the non-diffuser clocking scheme, the scheme $C L 4$, the effective value of outlet pressure pulsation and the amplitude at the main frequency were decreased by $27.2 \%$ and $35.8 \%$ respectively.

(2) The diffuser clocking enlarged the high pressure area of the last stage pump chamber and the pressurized water chamber of the multi-stage centrifugal pump and increased the static pressure value at the pump outlet. The diffuser clocking increased the head of the multi-stage pump to a certain extent.

(3) The diffuser clocking changed the shape of the vortices on the left and right sides of the impeller outlet of the multi-stage pump and reduced the vortex size. The vortices were no longer on the same horizontal line in the axial direction and the impeller outflow is smoother. The area and number of high 
turbulent kinetic energy in the outlet flow passage of the multi-stage centrifugal pump were reduced. Besides, the size of vortices in the flow passage was decreased, which made the outlet flow of the multistage pump more stable.

(4) The clocking effect of the diffuser optimized the uneven velocity at the inlet of the six diffuser passages and reduced the velocity gradient in the diffuser passages. The relative high-speed area at the outlet of the diffuser passages was also reduced.

(5) Compared with the non-diffuser clocking scheme, the clocking scheme CL4 reduced the hydraulic loss in the diffuser of the four pump stages by $2.43 \%, 12.15 \%, 11.43 \%$ and $13.19 \%$ respectively. The total reduction of hydraulic loss accounted for $1.11 \%$ of the head of the multi-stage pump.

\section{Declarations}

\section{Acknowledgements\&Funding}

The authors gratefully acknowledge the support of the following foundations: National Natural Science Foundation of China (No.51979124, 52179084, 51679110), and a project funded by the Priority Academic Program Development of Jiangsu Higher Education Institutions (PAPD).

\section{Availability of data and materials}

The datasets supporting the conclusions of this article are included within the article.

\section{Authors' contributions}

The author' contributions are as follows: Tan Ming-Gao was in charge of the whole trial; Sun Xue-Lei wrote the manuscript; Wu Xian-Fang assisted with sampling and Liu Hou-Lin assisted with laboratory analyses.

\section{Competing interests}

The authors declare no competing financial interests.

\section{Consent for publication}

Not applicable

\section{Ethics approval and consent to participate}

Not applicable

\section{Biographical notes}


Tan Ming-Gao, born in 1980, is a researcher and Ph.D. supervisor of Research Center of Fluid Machinery and Engineering Technology, Jiangsu University, China. His main research field is modern pump theoretical design and method.

E-mail:tmgwxf@ujs.edu.cn

Sun Xue-Lei, born in 1998, is a master candidate of Research Center of Fluid Machinery and Engineering Technology, Jiangsu University, China. His main research direction is the research on the internal flow mechanism of solid-liquid two-phase flow pump.

E-mail:2665304236@qq.com

Wu Xian-Fang, born in 1980, is an associate professor and master supervisor of School of Energy and Power Engineering, Jiangsu University, China. Her main research field is modern pump theoretical design and method.

E-mail:wxftmg@ujs.edu.cn

Liu Hou-Lin, born in 1971, is a researcher and Ph.D. supervisor of Research Center of Fluid Machinery and Engineering Technology, Jiangsu University, China. His main research field is modern pump theoretical design and method.

E-mail:liuhoulin@ujs.edu.cn

\section{References}

1. Dai H.W. Numerical simulation and experimental study on the effect of rotor timing on the performance of multistage centrifugal pump[D]. Master, Jiangsu University, Zhenjiang, China, 2015.

2. Chen F,Lu H.W,Gu Z.H,et al. One of the effects of axial clearance on compressor timing effect: total performance[J]. Journal of Engineering Thermophysics,2007,28(2): 232-234.

3. Yang H.T,Huang H.Y,Feng G.T,et al. Two-dimensional and three-dimensional numerical simulations of Clocking effect of stator blades in a low-speed compressor and verification of experimental results[J]. Journal of Engineering Thermophysics,2005,26(06): 927-930.

4. Yan P.G,Li S.B,Chen F,et al. Effect of timing effect of static Blade on unsteady aerodynamic load of Compressor Blade[J]. Journal of aeronautical power,2008,23(01): 117-124.

5. Walker G. J. Effect of wake-wake interactions on the generation of noise in axial-flow turbomachinery [C]. Institute de Mecanique des Fluides, International Symposium on Air Breaking Engines, 1 st st, Marseille, France, 1972.

6. Saren V E. Some Ways of Reducing Unsteady Loads Due to Blade Row Hydrodynamic Interaction in Axial Flow Turbomachines [C]. Second International Conference EAHE, Pilsen, Czech Republic, 1994: 160-165. 
7. Cizmas P, Subramanya R. Parallel computation of rotor-state interaction [M]//Unsteady Aerodynamics and Aeroelasticity of Turbomachines. Springer Netherlands, 1998: 633-645.

8. Mu-ting H, Rong X, Liang G. Clocking Effect on Unsteady Aerodynamic Loading on the Blade in the Centrifugal Compressor [J]. Compressor,Blower \& Fan Technology, 2016, 58 (1): 15-21.

9. Geng X. Study on hydraulic characteristics of intake Mode of Primary Impeller of Multi-stage pump[D]. Master,Huazhong University of Science and Technology, Wuhan, China, 2009.

10. Zhang K. Study on optimal Design and cavitation performance of Multi-stage centrifugal pump[D]. Master, Huazhong University of Science and Technology, Wuhan, China, 2014.

11. Fu J.G. Analysis of fluid-solid Coupling dynamic characteristics of Multi-stage centrifugal pump[D]. Master, Yangzhou University, Yangzhou, China, 2014.

12. Liu J.X. Optimal selection and Energy Saving Retrofit of Boiler Multi-stage pump[J]. Metallurgical energy, 2013, (5): 57-58.

13. Li X.G, Yuan M.Q, Cheng A.H. Optimal Design of Axial channeling Control Mode of Segmental Multistage pump[J]. Pump technology, 2012, 13(5): 5-7.

14. Benigni $H$, Jaberg $H$, Yeung $H$, et al. Numerical Simulation of Low Specific Speed American Petroleum Institute Pumps in Part-Load Operation and Comparison With Test Rig Results [J]. Journal of Fluids Engineering, 2012, 134 (2): 130-130.

15. Fu H, Lu J.L, Chen N, et al. Numerical Simulations on Clocking Effect of Impeller-stator in Multi-stage Axial-flow pump [J]. Journal of Drainage and Irrigation Mechanical Engineering, 2016, 34 (11): 934940.

16. Ye C.L, Wang F.J, Li H.C, et al. Investigation on the Clocking Effect of a Double-entry Two-stage Double Suction Centrifugal Pump with High Head[J]. Journal of Hydraulic Engineering, 2018, 49 (7).

17. Zhu X.Y, Jiang W, Li G.J, et al.Numerical Investigation of Influence of Installation Location between Diffuser Vane and Volute Tongue on Performance of Single-stage Centrifugal Pump [J]. Engineering Journal of Wuhan University, 2018, 51 (01): 72-79.

18. Yao Z.F, Wang F.J, Yang M, et al. Effects of Impeller Type on Pressure Fluctuations in Double-suction Centrifugal Pump [J]. Journal of Mechanical Engineering, 2011, 47 (12): 133-137.

19. Lu J.L, Guo P.C, Wang L.K, et al. Influence of Impeller Clocking on the Inner Flow of A Two-stage Centrifugal Pump [J]. Journal of Xi'an University of Technology, 2019, 35 (03): 300-307.

20. Zhang K.K, Tan M.G, Wu X.F, et al. Clocking effect of guide vane in multi-stage centrifugal pump[J]. Journal of drainage and irrigation machinery engineering, 2021, 39(7): 663-670.

21. Zhang Q, Yan X, Shi W, et al. Design and performance test of circumferential crankle guide vane of multistage centrifugal pumps[J]. Transactions of the Chinese Society of Agricultural Engineering, 2013, 29(5): 37-43.

22. Lugovaya S, Olshtynsky P, Rudenko A, et al. Revisited Designing of Intermediate Stage Guide Vane of Centrifugal Pump[J]. Procedia Engineering, 2012, 39: 223-230. 
23. Kong F.Y,Su X.H,Chen H,et al. Optimal Design of positive Vane parameters of diameter Guide Vane of centrifugal pump[J]. Journal of agricultural engineering. 2012,28(23): 40-45.

24. Wang J.Q,Kong F.Y. Study on hydraulic performance Optimization of Impeller and Guide Vane of Multi-stage centrifugal pump[J]. Journal of Huazhong University of Science and Technology (Natural Science). 2013,41(03): 2-96.

25. Alireza F., Arno M., Michael T. (2018) Accuracy and comparison of standard k- $€$ with two variants of k- $\omega$ turbulence models in fluvial applications. Engineering Applications of Computational Fluid Mechanics. 12(1), 216-235.

\section{Figures}

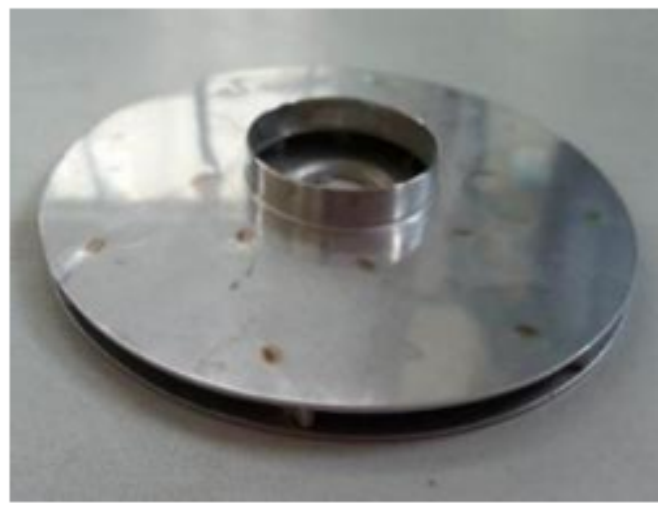

(a)Impeller

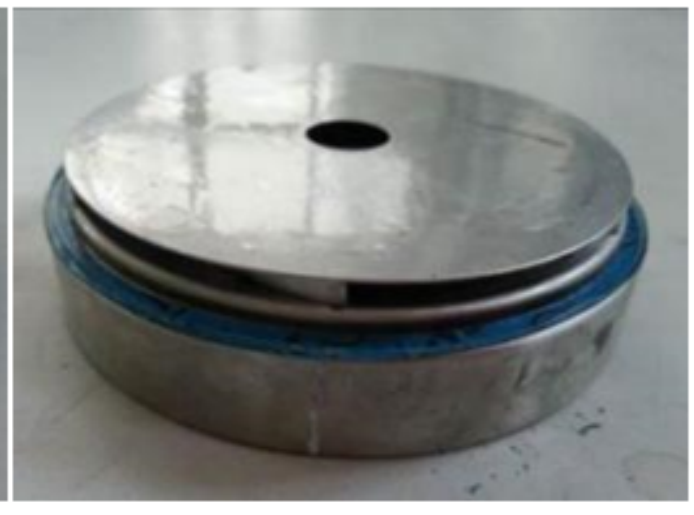

(b)diffuser

\section{Figure 1}

Segmental type 5-stage centrifugal pump

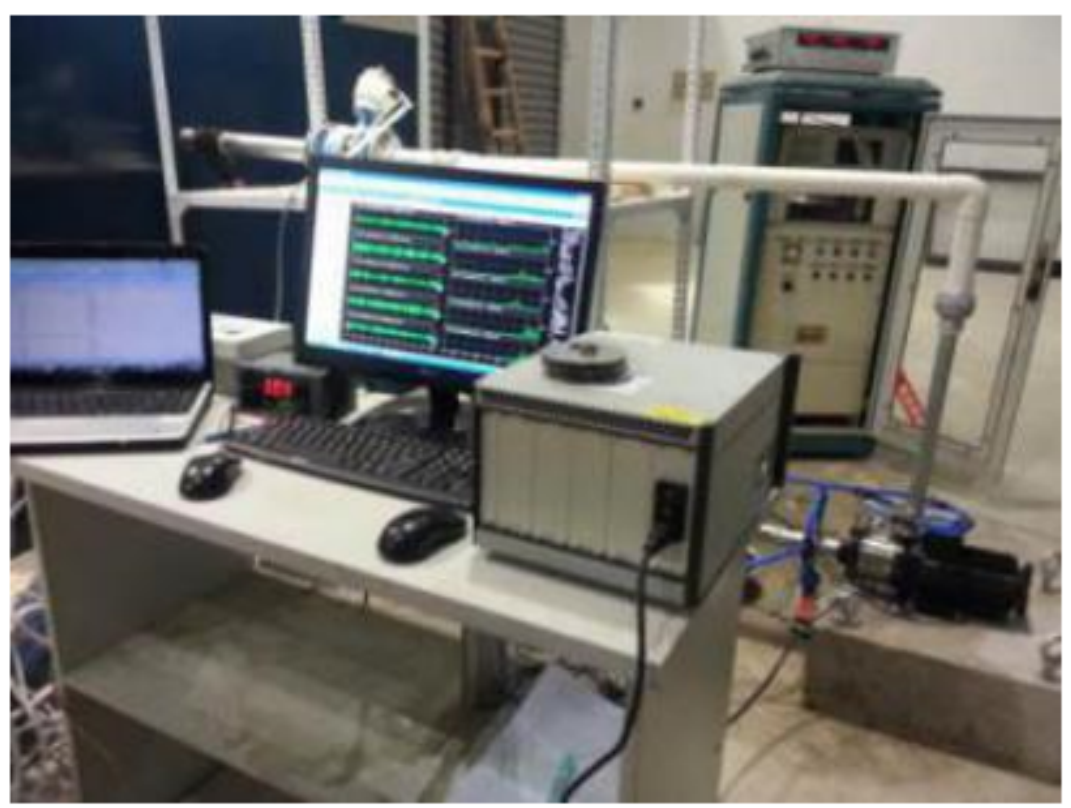


Figure 2

Multi-stage pump synchronous test table

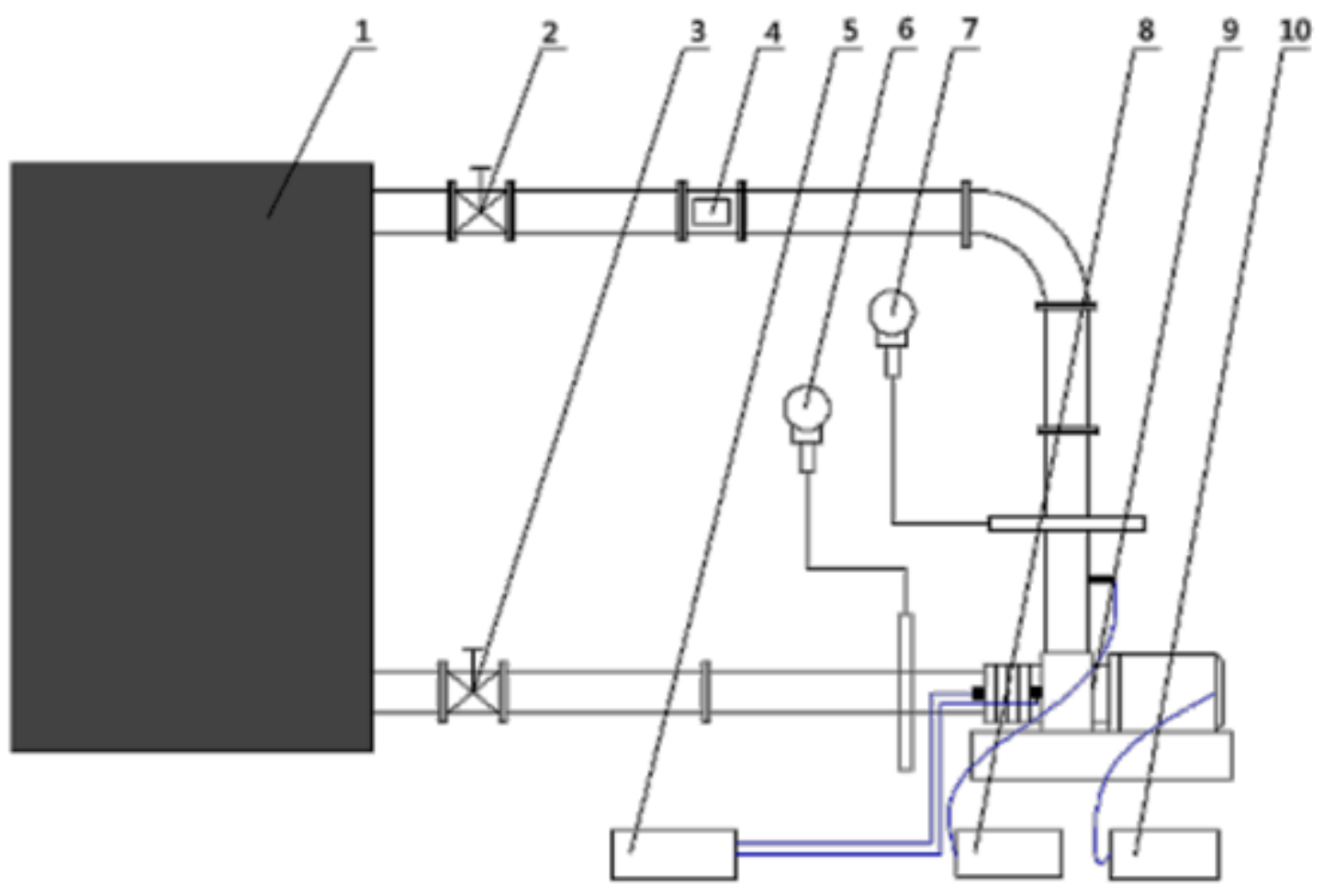

Figure 3

Experimental device 1.water tank 2. outlet valve 3. inlet valve 4. flowmeter 5.vibration signal and sensor 6. inlet pressure transmitter 7. inlet pressure transmitter 8. pulse signal collector and sensor 9 . multistage pump 10. frequency converter

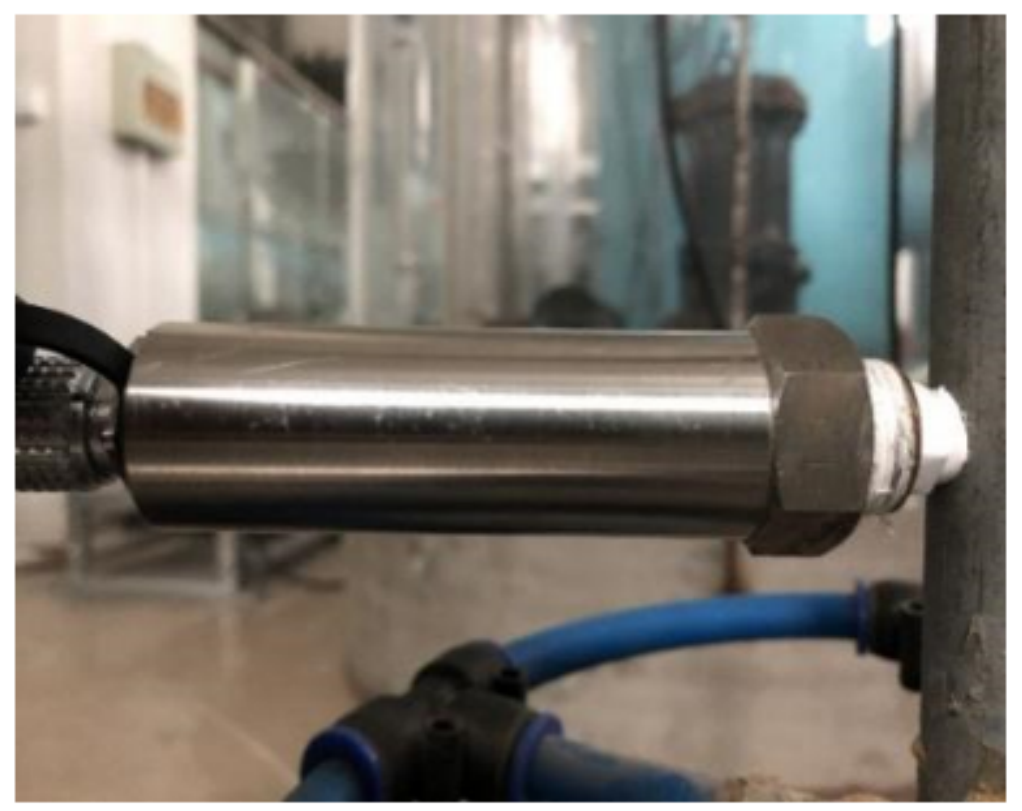


Figure 4

Outlet pulsation sensor layout

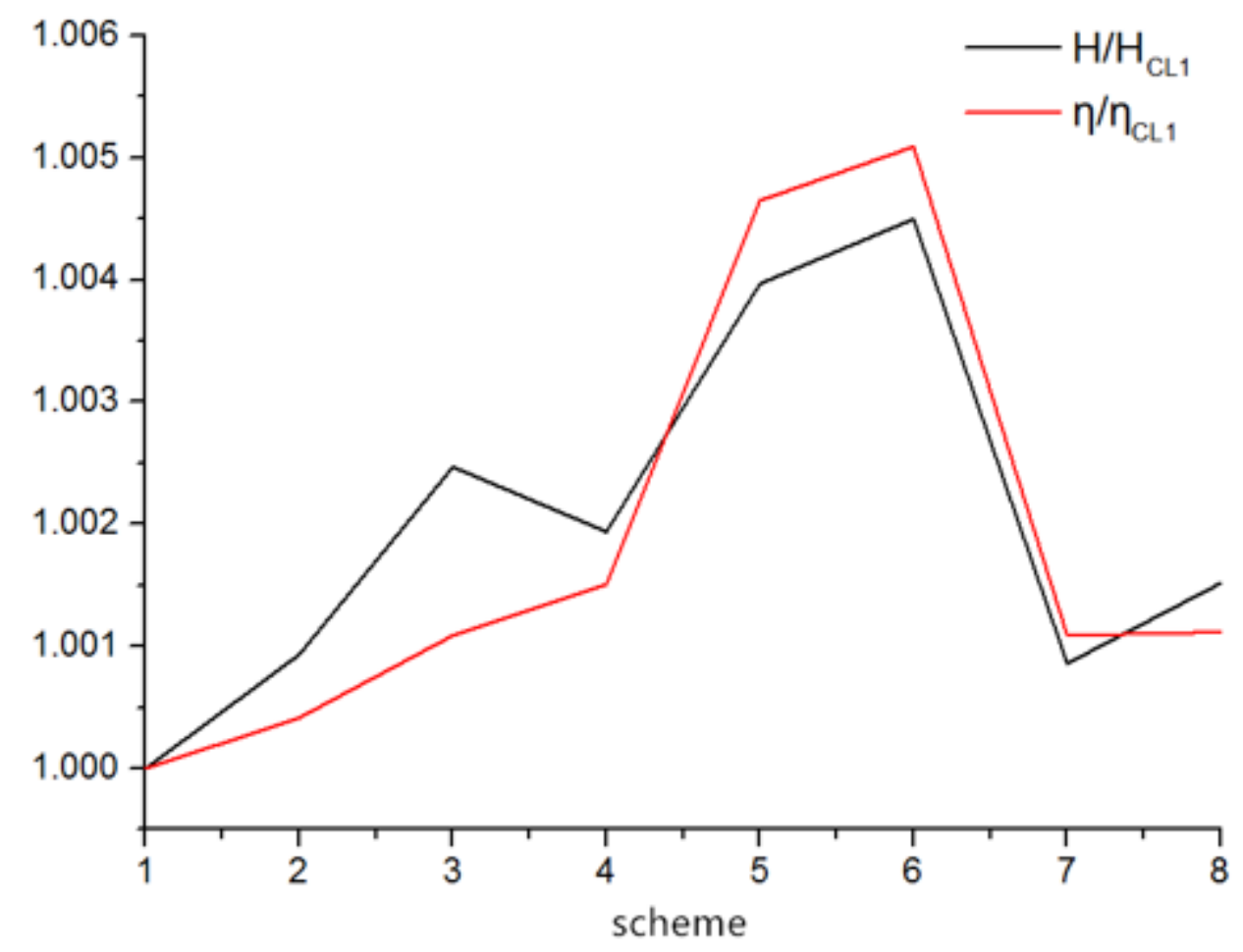

Figure 5

Comparison of Head and Efficiency

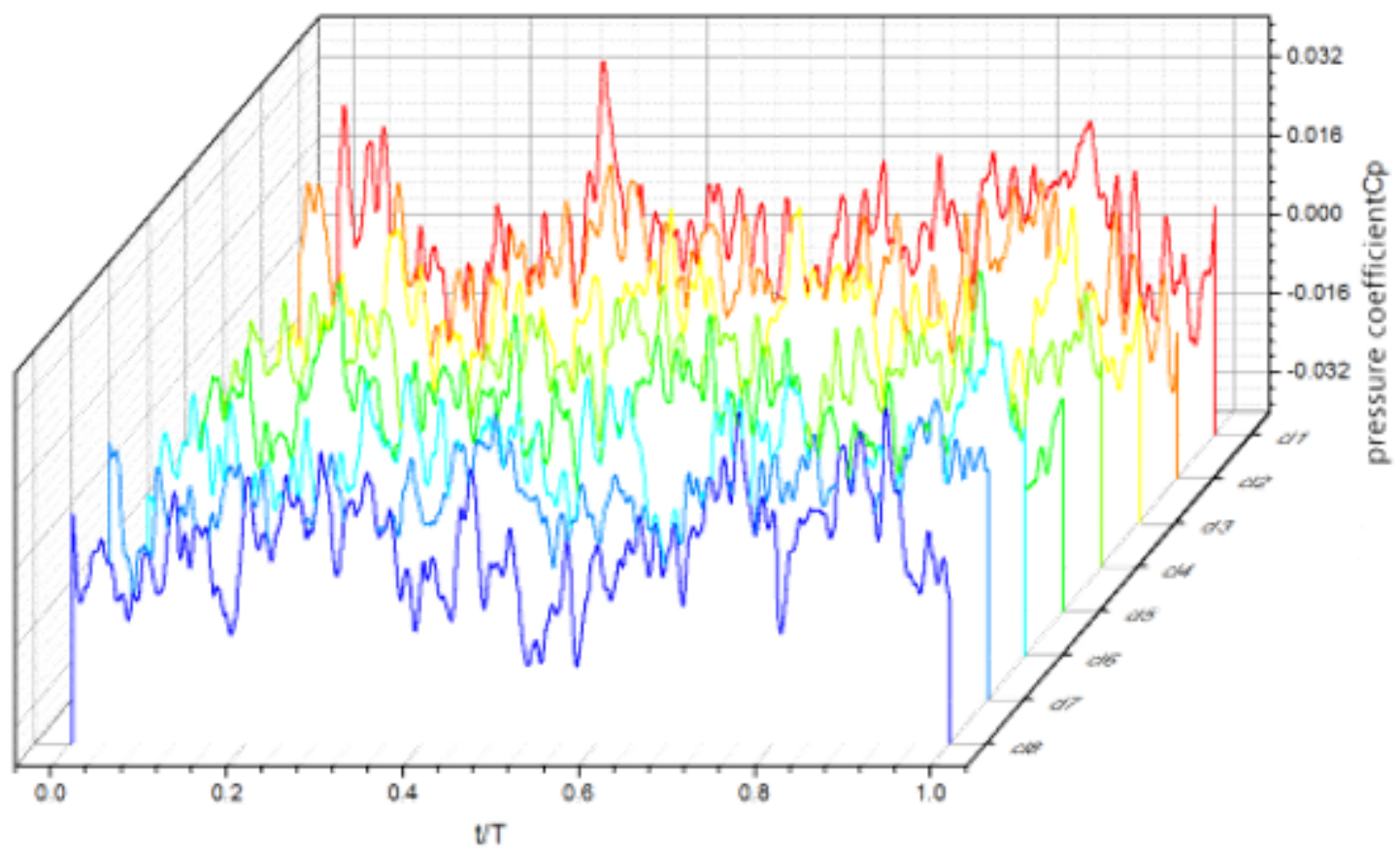

Figure 6 
Time domain diagram of outlet pressure coefficient

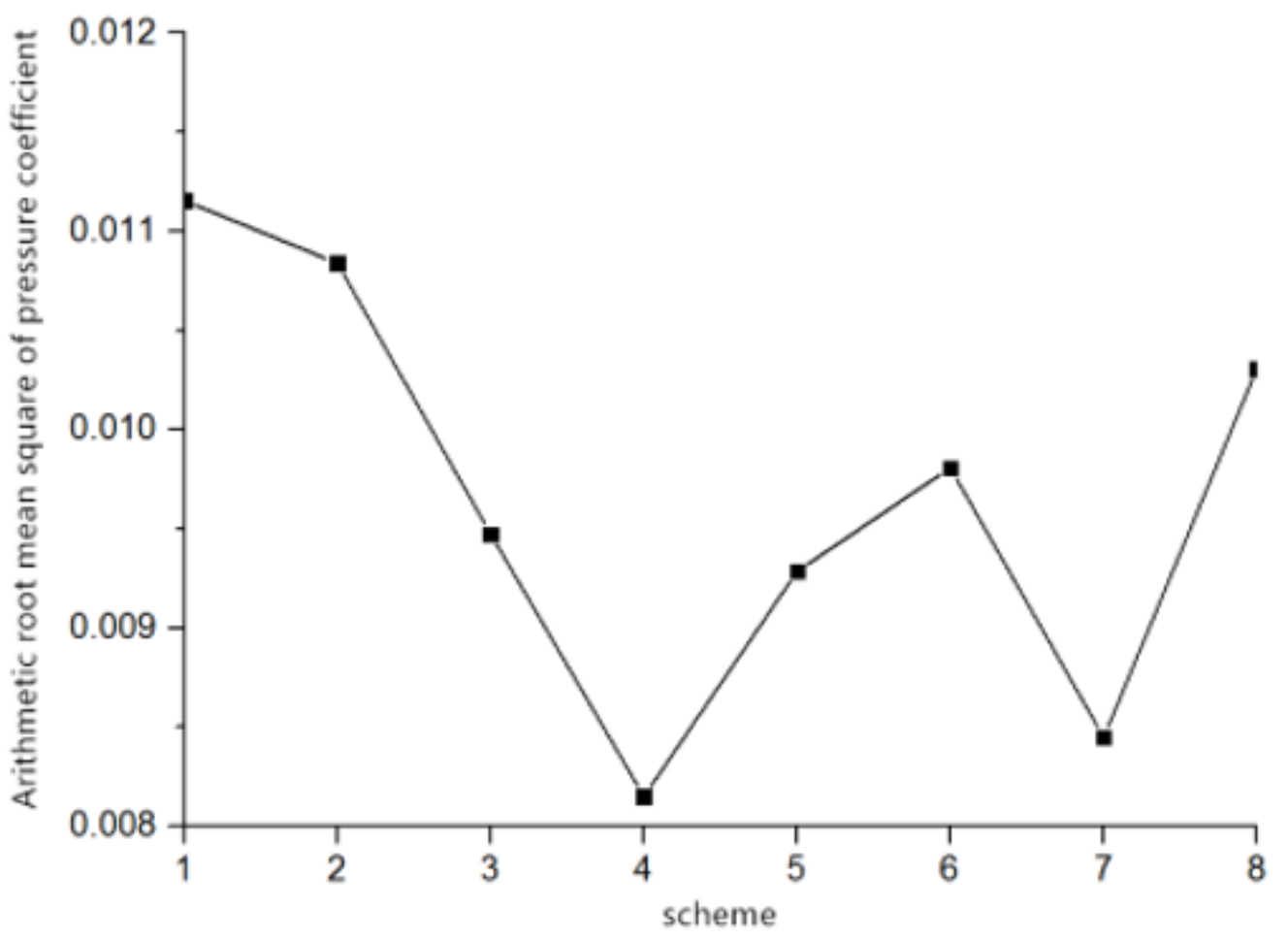

Figure 7

Arithmetic root mean square of pressure coefficient

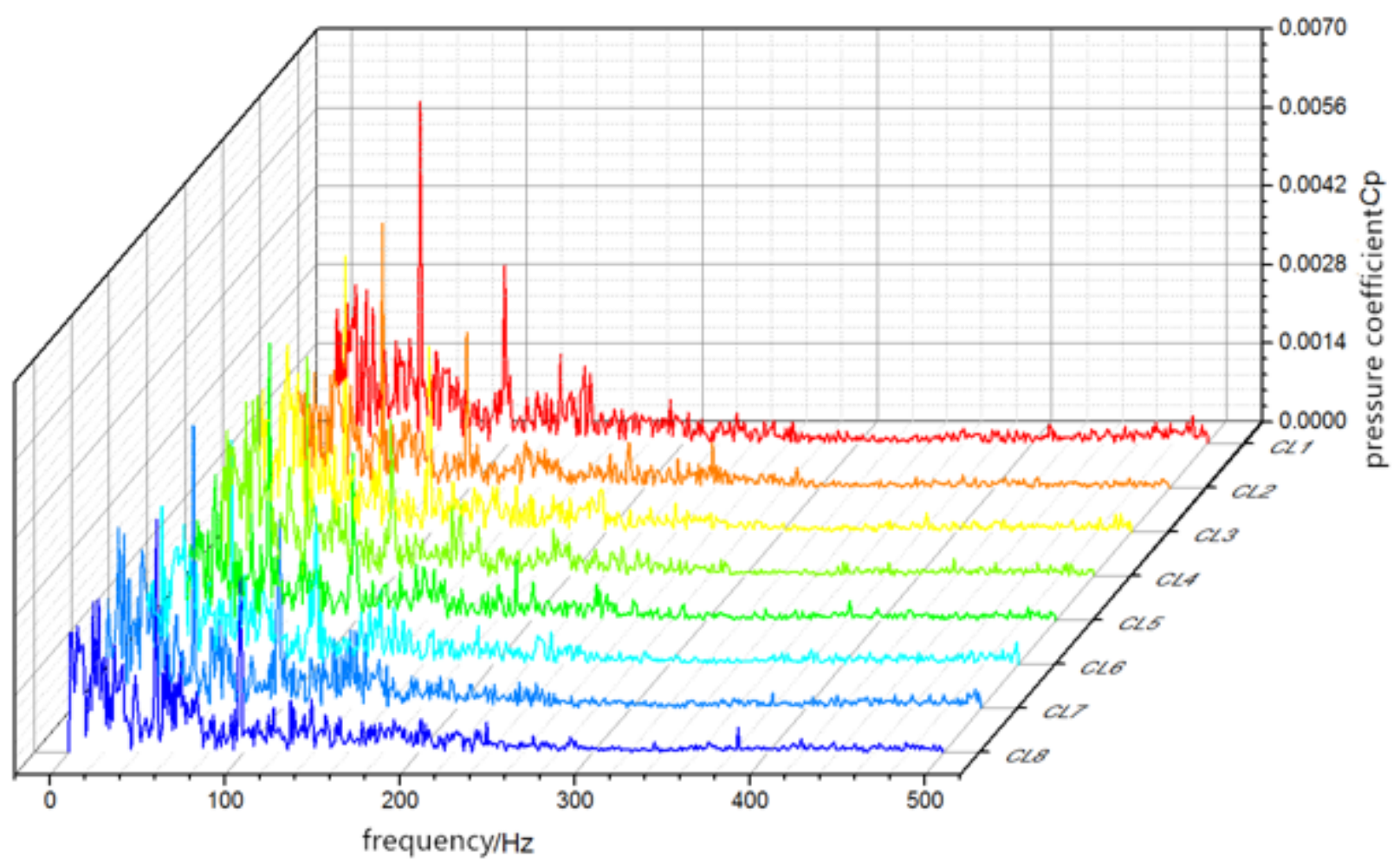

Figure 8 
Frequency domain distribution of pressure coefficient

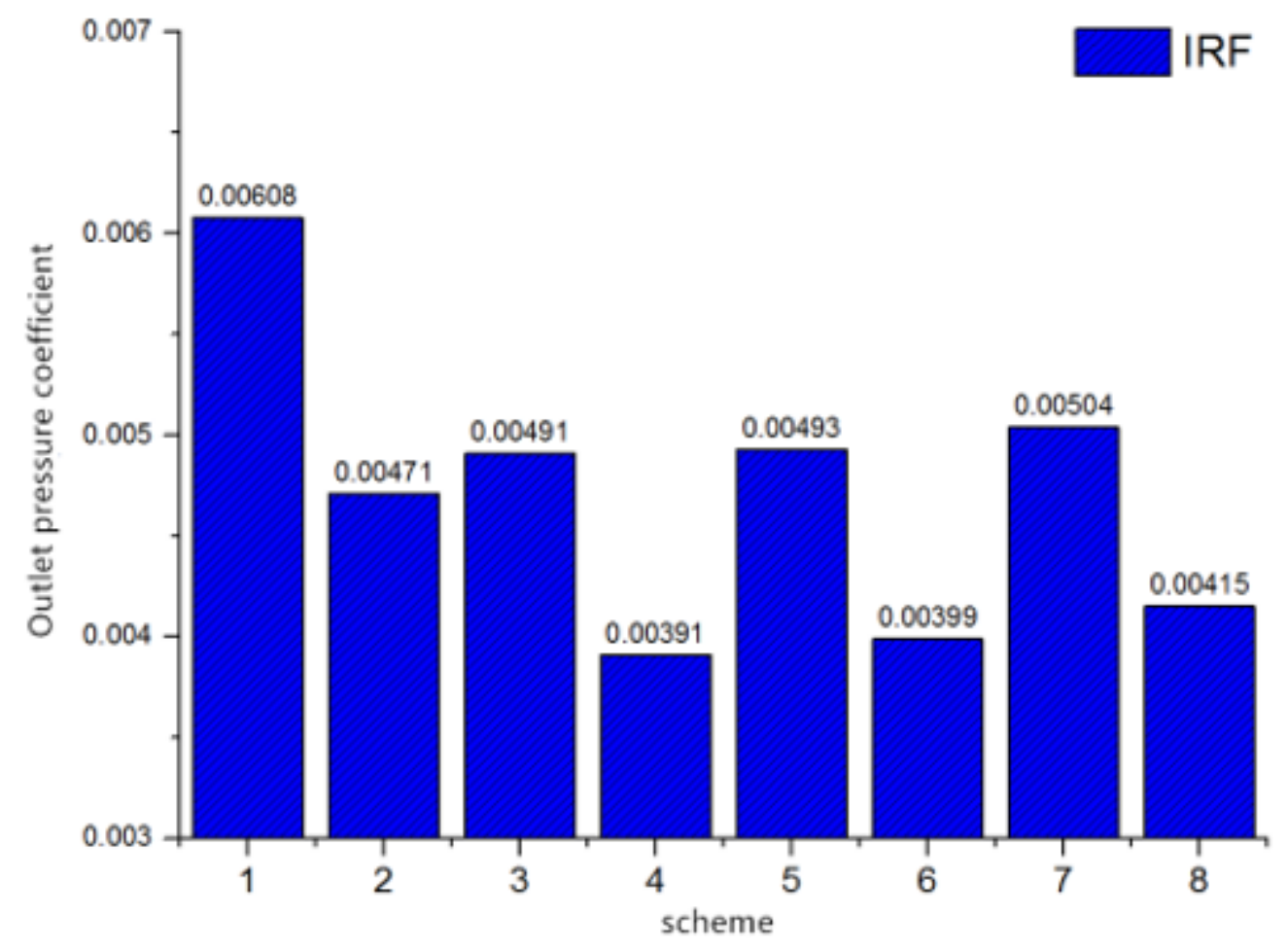

Figure 9

Dominant Frequency Amplitude of Outlet Pressure Coefficient for Each Scheme

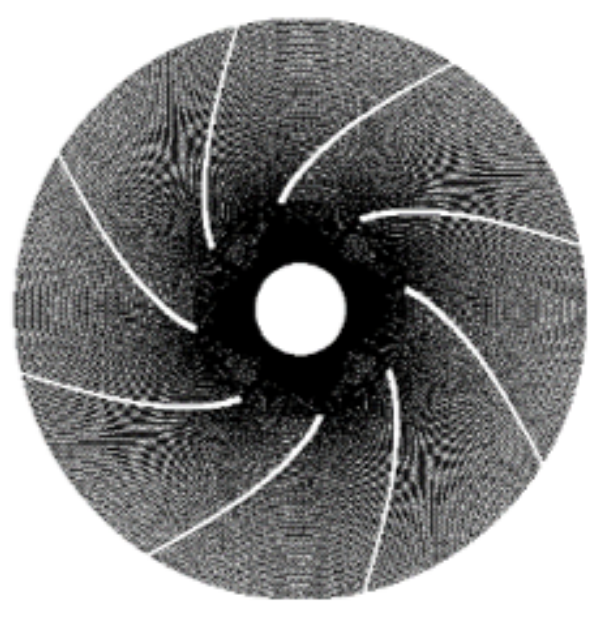

(a)Impeller

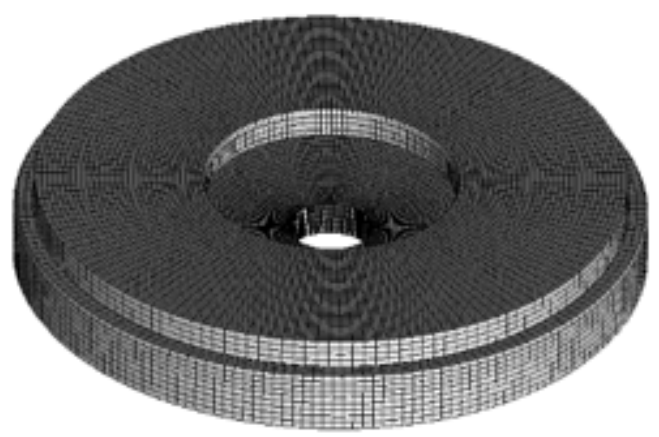

(b)diffuser

Figure 10

Structured Mesh Computing Domain 


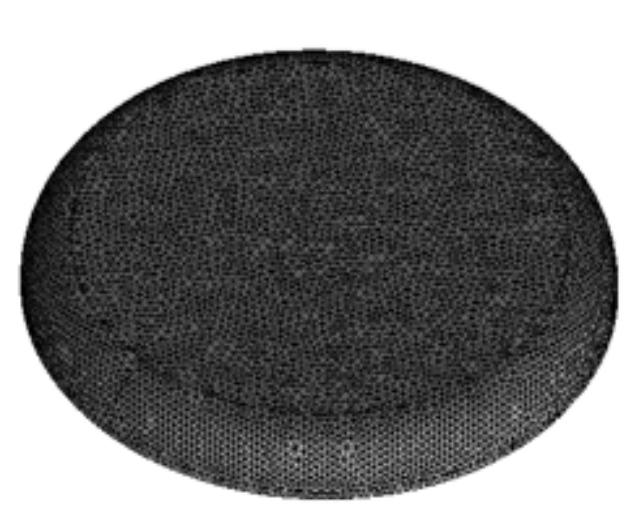

(a) First Pump Chamber

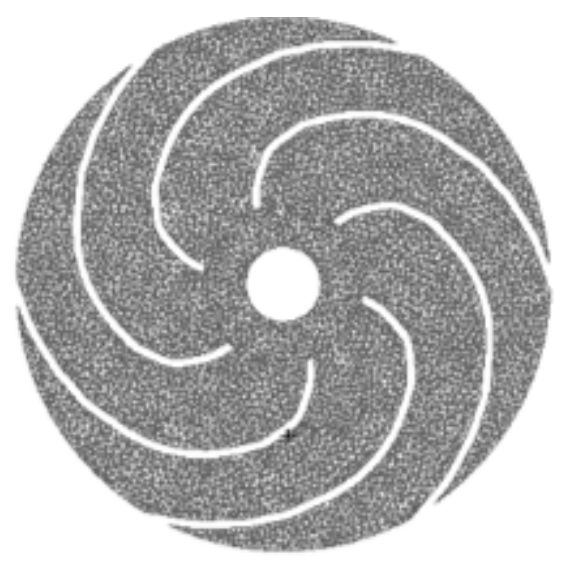

(b) Diffuser

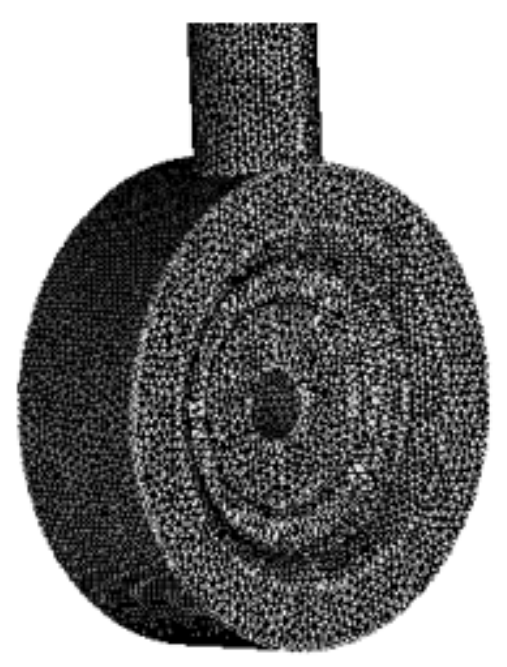

(c) Last water pressure chamber

Figure 11

Unstructured Mesh Computing Domain

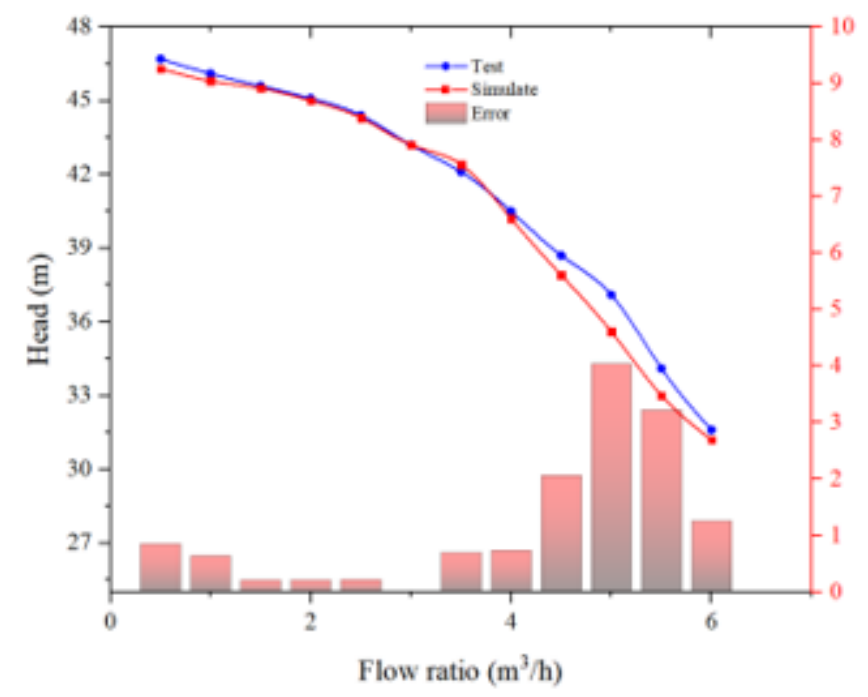

(a)Head

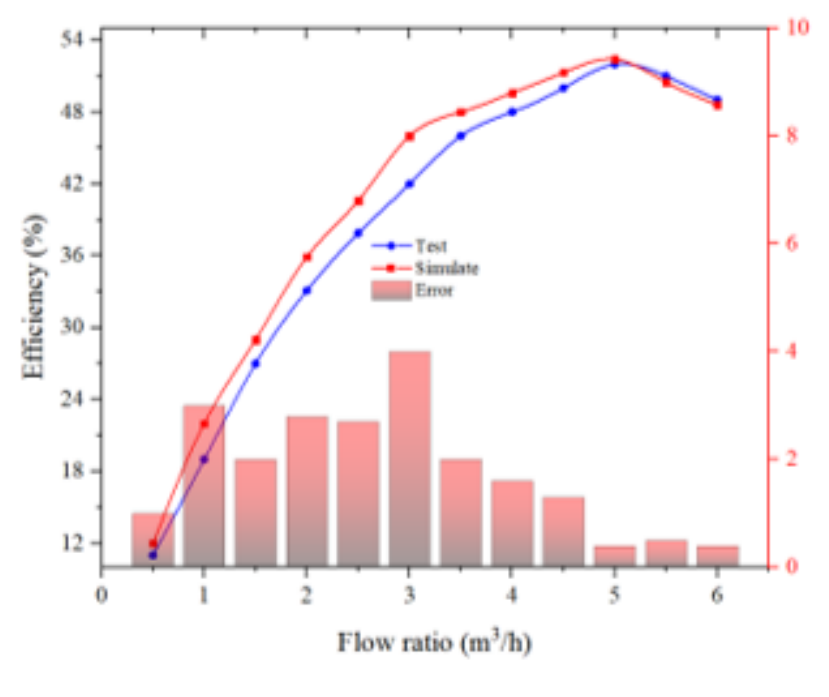

(b) Efficiency 
Figure 12

A comparison $\left(\varphi=0^{\circ}\right)$ between the simulated predicted value and the test value of the head and efficiency

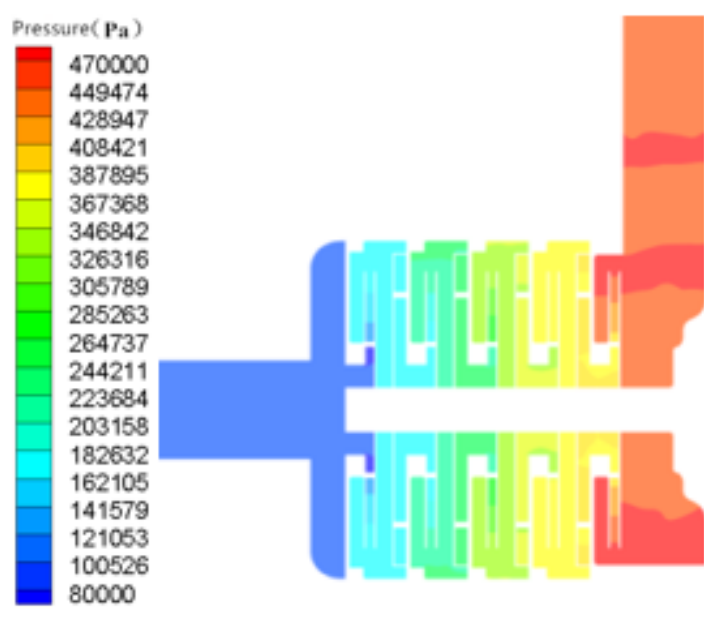

(a)CL1

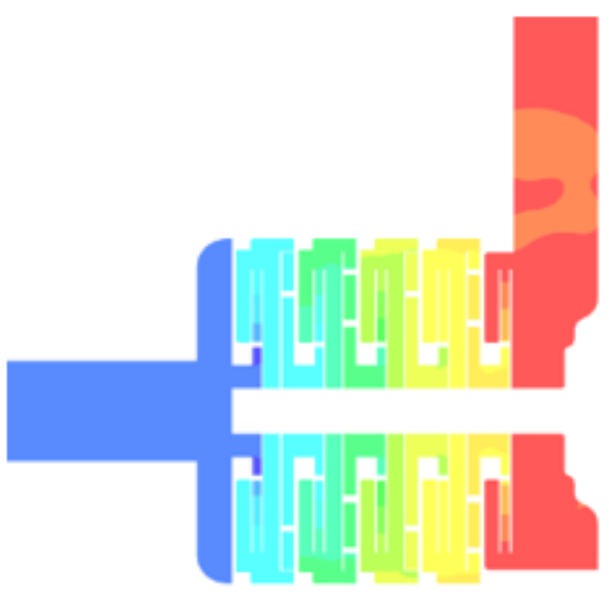

(b)CL4

Figure 13

Static pressure distribution 

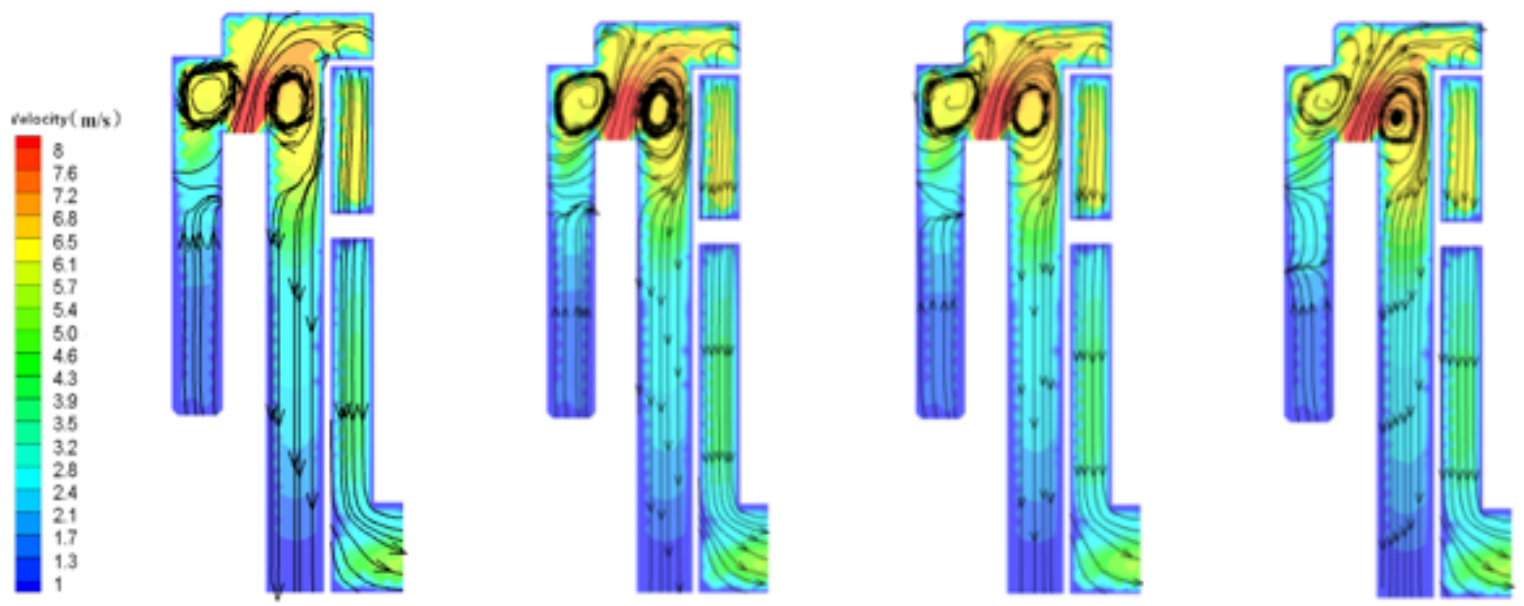

CL1:
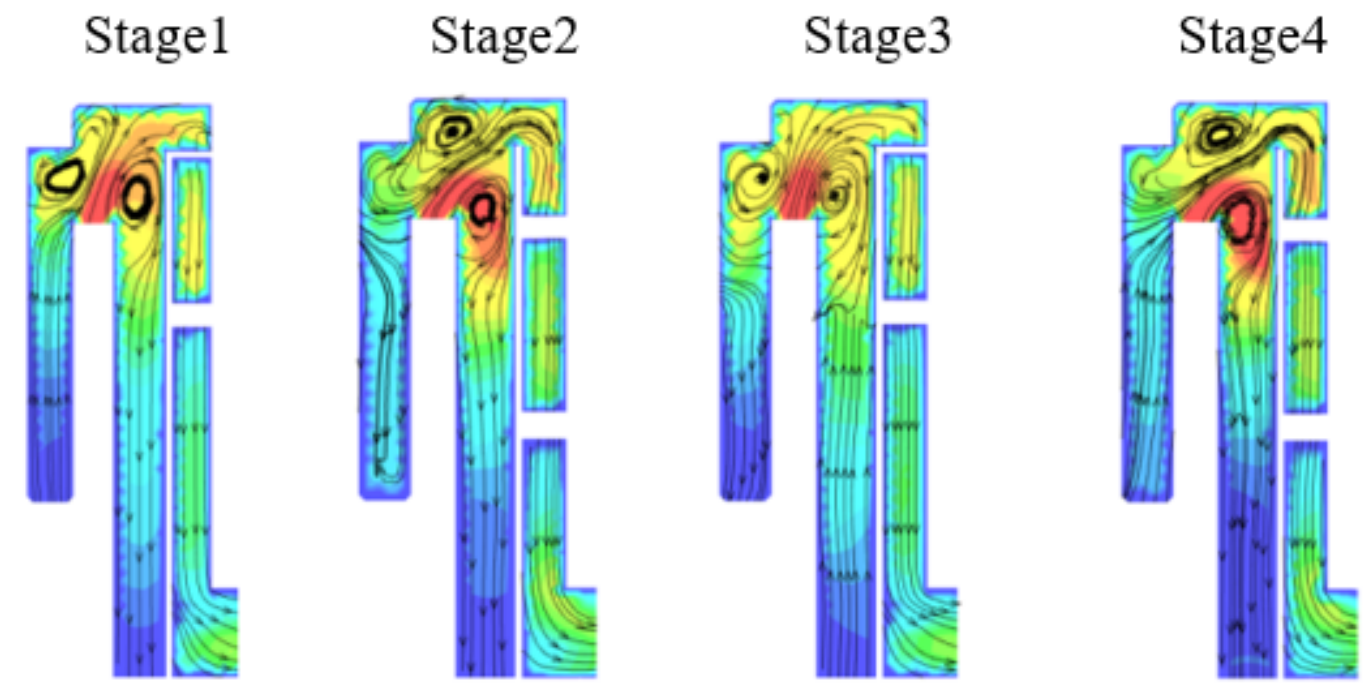

CL4:

Stage1

Stage2

Stage3

Stage4

Figure 14

Axial velocity of pump chamber of each pump stage 

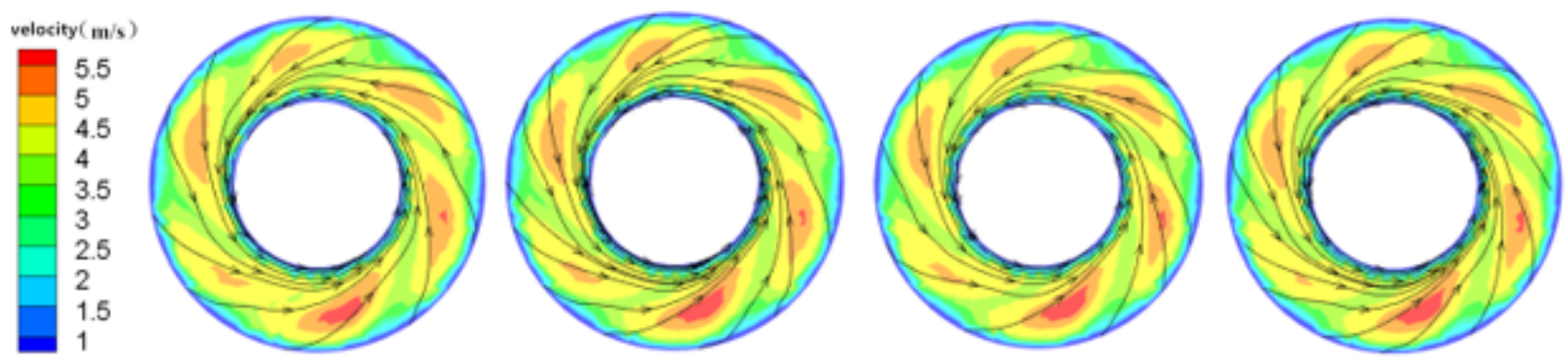

CL1 : Stage1

Stage2

Stage3

Stage4
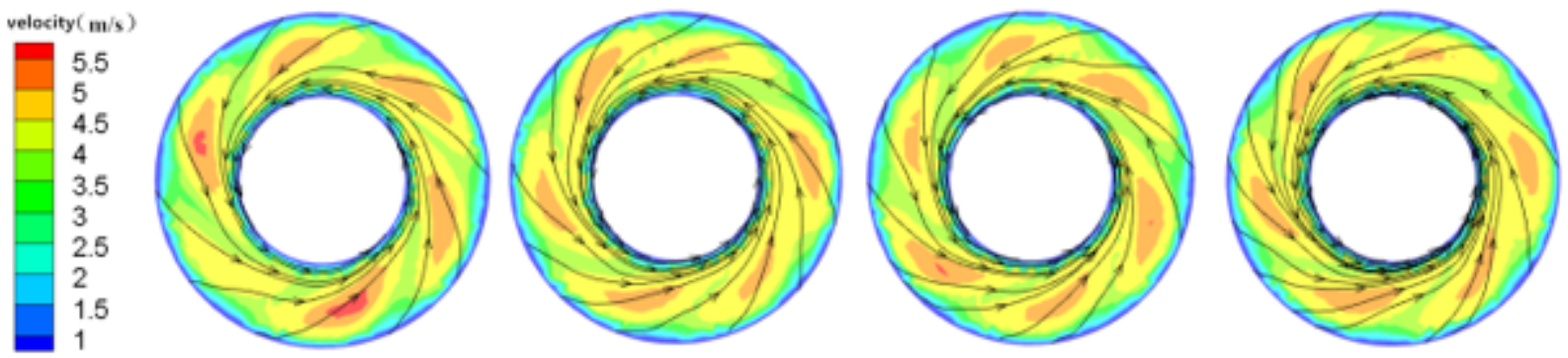

CL4: Stage1

Stage2

Stage3

Stage4

Figure 15

Velocity Distribution of diffuser Outlet Section

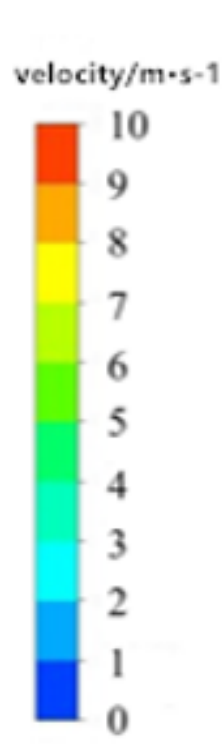

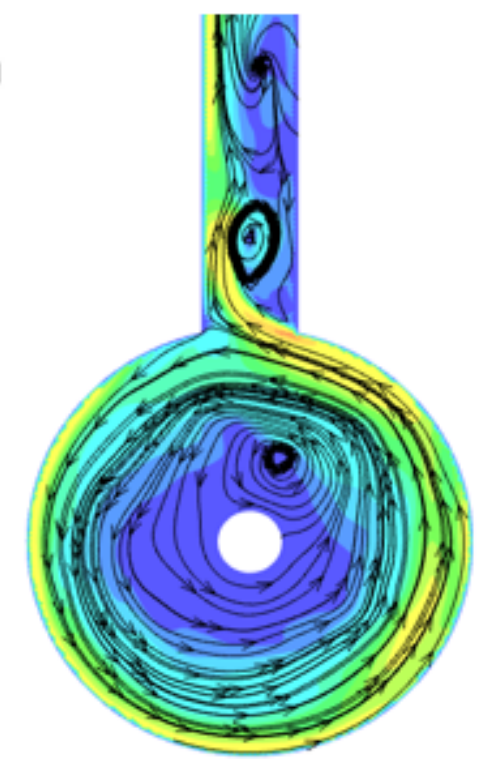

(a)CL1

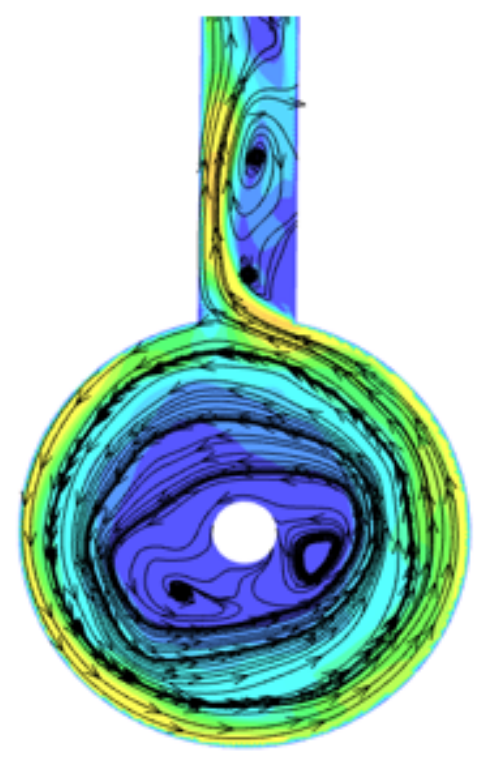

(b)CL4

Figure 16

Distribution of Planar Velocity 


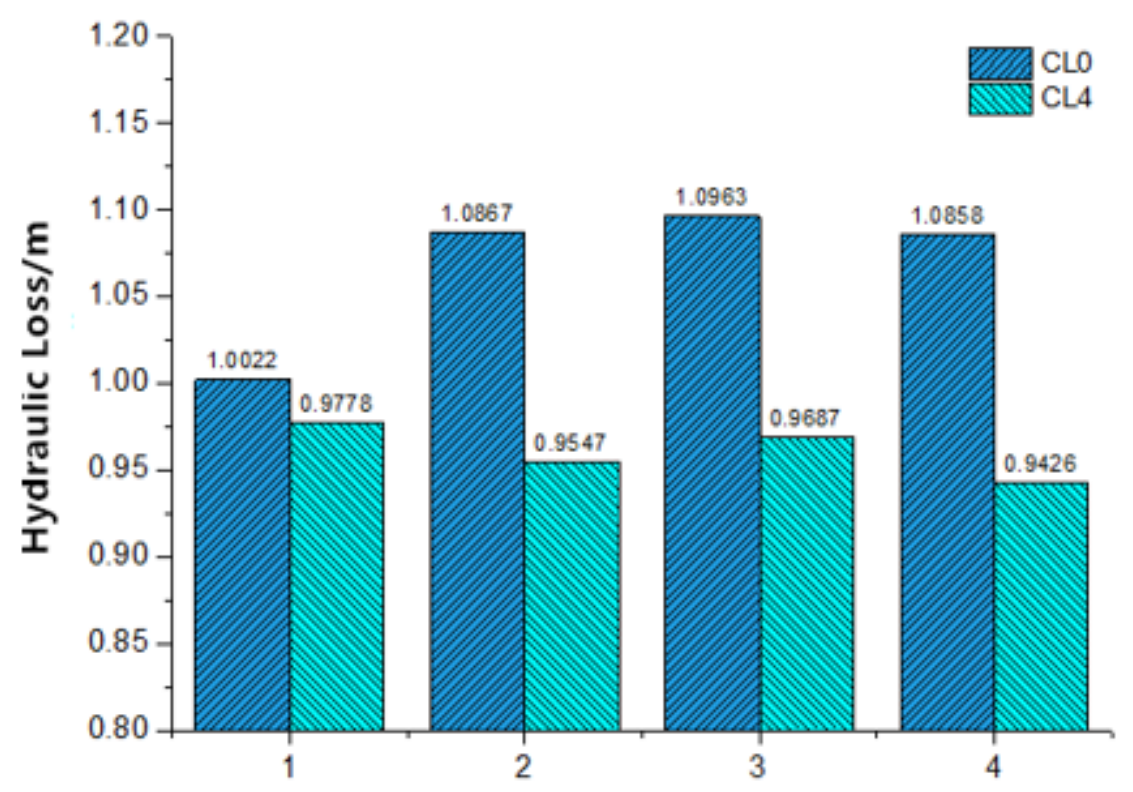

Figure 17

Hydraulic Loss of diffuser Flow Channel

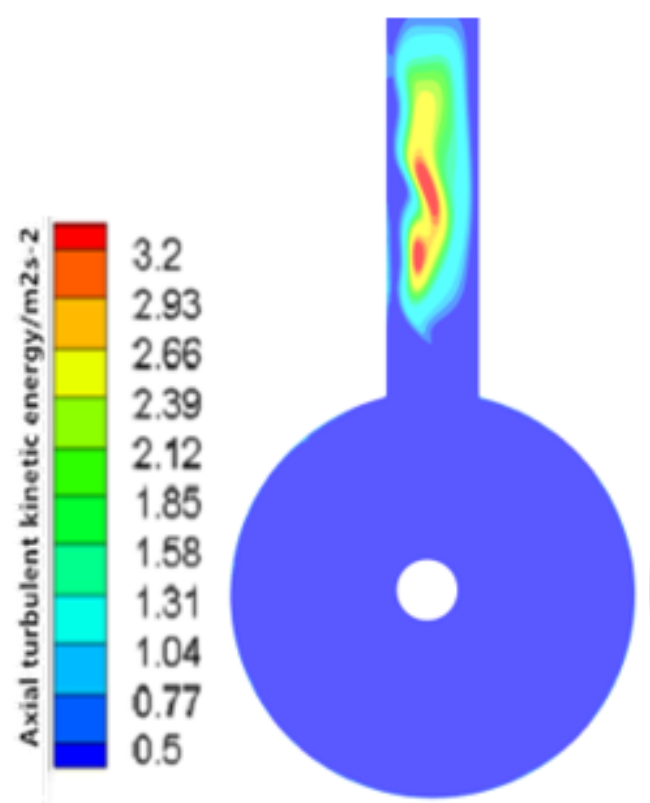

(a)CL1

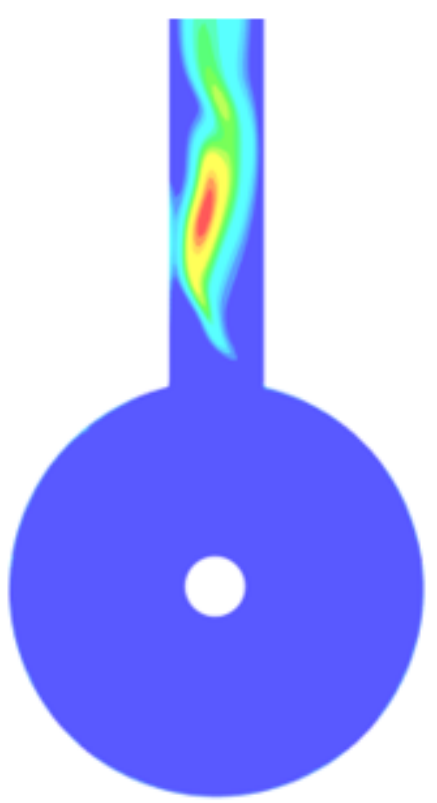

(b)CL4

Figure 18

Axial turbulent kinetic energy distribution 